\title{
Are Areas of Isolated Atypical Cells or Residual Tumor Cartilage Prognostically Significant in Neoadjuvant Treated Osteosarcoma? An Analysis of 106 Consecutive Cases from a Single Institution
}

\author{
Michael J. Cascio ${ }^{1}$, Richard J. O’Donnell ${ }^{2}$, Robert E. Goldsby ${ }^{3}$ and Andrew E. Horvai* ${ }^{*}$ \\ ${ }^{I}$ Department of Pathology, ${ }^{2}$ Department of Orthopaedic Surgery, and ${ }^{3}$ Department of Pediatrics, University of \\ California San Francisco, San Francisco, CA, USA
}

\begin{abstract}
Treatment of high-grade osteosarcoma consists of neoadjuvant chemotherapy then surgical resection. Generally, < $90 \%$ tumor necrosis predicts an unfavorable prognosis and can influence post-operative therapy. Thus, accurate, reproducible, microscopic measurement of tumor necrosis is imperative. Treated osteosarcomas contain a variety of morphologic patterns making quantitative assessment difficult. Whether residual tumor cartilage or widely dispersed atypical cells in fibrous stroma represent prognostically significant "viable" tumor is unknown and, to our knowledge, has not been formally tested. Full cross sections of the post-chemotherapy resection specimens of 106 osteosarcomas were evaluated for the presence and pattern of viable tumor using two methods. First, we counted as viable only dense areas of mitotically-active atypical cells ("stringent"). Second, we also included as viable rare (<25 / 400X field), atypical cells in fibrous stroma and/or tumor cartilage ("inclusive"). Treatment response was judged as good ( $\geq 90 \%$ necrosis) or poor ( $<90 \%$ necrosis) and correlated with disease-free survival using the Kaplan-Meier method. Eleven tumors (10\%) had no evidence of residual tumor after chemotherapy. The remainder contained different proportions of tightly-packed, atypical, mitotically-active cells $(n=73,69 \%)$, isolated atypical cells in fibrosis or necrosis $(n=35,33 \%)$ and/or tumor cartilage $(\mathrm{n}=10,8 \%)$. Disease-free survival correlated significantly with chemotherapy response using the "stringent" method $(\mathrm{p}=0.008)$ but not the "inclusive" method $(\mathrm{p}=0.34)$. These data confirm that the percent tumor necrosis after chemotherapy is an important prognostic indicator, but areas of tumor cartilage or widely distributed, mitotically-inactive, atypical cells should not be measured as residual viable tumor.
\end{abstract}

Keywords: Osteosarcoma, chemotherpay, viability.

\section{INTRODUCTION}

Osteosarcoma (OS) is the most common primary nonhematopoietic malignancy of bone. While relatively uncommon, the incidence of OS is greatest in the adolescent and young adult population at 4.4 cases per million per year, with a second peak in adults over the age of 60 years [1]. Conventional OS is defined microscopically by malignant cells associated with osteoid production. Various histopathologic subtypes have been described, including osteoblastic, chondroblastic, fibroblastic, small cell and telangiectatic although there is no current established relationship between subtype and prognosis [2].

Regardless of histologic subtype, current treatment for conventional high-grade OS includes 8-10 weeks of neoadjuvant chemotherapy, typically with MAP (methotrexate, doxorubicin, cisplatin) with leucovorin rescue. Neoadjuvant therapy allows assessment of treatment response, provides opportunity for surgical planning and, depending on the response, may allow limb-salvage in some who would require an amputation without neoadjuvant

*Address correspondence to this author at the Department of Pathology, University of California, San Francisco, 1600 Divisadero Street B220, San Franisco CA 94115, USA; Tel: (415) 885-1613;

Fax: (415) 673-9726; E-mail: Andrew.Horvai@ucsf.edu therapy [3-5]. Additional adjuvant chemotherapy is administered after definitive surgery. This approach has yielded 5-year survival rates of $70 \%$ for patients with localized disease compared to historical cure rates of $10-20 \%$ when using surgical resection alone [6, 7]. In resection specimens of treated OS, histologic response to chemotherapy has repeatedly been shown to predict improved survival. Consequently, a variety of qualitative and quantitative methods have been proposed to grade chemotherapy response histologically $[4,5,8-15]$, but the most widely used system accepts $\geq 90 \%$ tumor necrosis after chemotherapy as a "good" response that subsequently predicts favorable outcome [9, 14]. The adequacy of histologic response affects the adjuvant chemotherapy regimen. Therefore, the surgical pathologist must meticulously evaluate treated osteosarcoma specimens to quantify tumor necrosis. However, several variables render practical, reproducible assessment of tumor necrosis a challenge and make this process semi-quantitative, at best.

Most importantly, the diverse morphologic patterns of osteosarcoma after chemotherapy make the distinction between viable and necrotic areas subjective and inherently challenging. Initial descriptions proposed grading treatment response based on the proportion of "acellular tumor osteoid, necrotic or fibrotic material" to "histologically viable tumor" $[8,16]$. Sheets of malignant cells resembling the tumor in 
pretreatment biopsy or acellular osteoid can be reliably classified as viable and necrotic, respectively. However, "individual tumor cells in chemotherapy-responsive stroma" or "atypical cells with hyperchromatic nuclei, smudged and clumped chromatin and vacuolated cytoplasm in a background of necrosis, calcification or fibrosis" are more difficult to classify $[17,18]$. It is unclear whether such cells neoplastic. Furthermore, should residual viable neoplastic cartilage with atypical chondrocytes be included in the measurement of viable tumor? Some have recommended to interpret the smudgy atypical cells as "viable"[17] but this is not the practice at all institutions, including our own. The approach by some pathologists is to score only those areas of tumor that resemble the osteogenic component of the pretreatment biopsy (AEH, personal communication) as "viable." The practice reflects the uncertainty of the biological potential of isolated cells with smudgy chromatin and the inherent difficulty quantitating the area occupied by individual tumor cells or chondrocytes amid abundant stroma. But, from a practical perspective, the question for the pathologist is not whether such cells are viable in a biological sense but whether areas containing isolated tumor cells or tumor cartilage, if measured as viable, are prognostically significant. To our knowledge, this question has not been formally tested.

Superimposed on the above challenges is the limited number of high-quality color photomicrographs in the literature depicting exactly what comprises viable or necrotic tumor [5, 16, 18-20]. Furthermore, pre-resection CT scans and angiograms are not always available to determine the optimal sampling, as suggested by some protocols [5, 17], but sectioning based on gross impressions [15] may not be representative. Finally, residual viable tumor often presents as multiple, irregularly shaped nodules. Can these areas be estimated with simple formulas and remain prognostically significant or are less practical and more time consuming methods necessary?

The aims of this study were to describe the histomorphologic patterns of chemotherapy response in patients with osteosarcoma and to assess whether areas of residual tumor cartilage and/or individual tumor cells in fibrous stroma should be included in the measurement of viable tumor.

\section{MATERIALS AND METHODS}

The study was carried out according to guidelines set forth by the Committee on Human Research of the University of California, San Francisco. We retrospectively identified cases of osteosarcoma in the pathology archives at our institution between 1996 and 2012. A total of 132 cases were identified, of which 26 were excluded because they were not treated with neoadjuvant therapy, post treatment resection specimens were unavailable and/or were surface types of osteosarcoma (parosteal and periosteal). Thus, 106 consecutive cases of conventional high-grade, intramedullary osteosarcoma were available for study. The diagnosis and subtype of osteosarcoma was based on morphologic and radiographic evaluation of the pre-treatment biopsies, according to the World Health Organization criteria [19].

For each case, the age of the patient at the time of diagnosis, anatomic location of the tumor, tumor subtype, and type of resection, duration of clinical follow-up and presence of recurrence and/or metastasis was recorded. The post-chemotherapy resection or amputation specimens were processed as previously described [15]. Specifically, after taking sections of the soft tissue for margin evaluation, full cross sections were made, usually in the sagittal or coronal planes, to produce a single $0.5 \mathrm{~cm}$ slice or slab of maximal tumor cross-sectional area. The tumor area was estimated using the product of the maximum length and width of gross tumor on the slice. Locations of sections taken for histologic examination were then recorded on a photograph or photocopy of the slice (Fig. 1).

Hematoxylin and eosin-stained sections were evaluated histologically by MJC and $\mathrm{AEH}$, who were blinded to clinical outcome, for the presence and pattern of viable tumor. On each glass slide, areas containing viable tumor were outlined (Fig. 1). Cross sectional areas were estimated using the formula for a rectangle (maximum length $\mathrm{x}$ maximum width) regardless of the shape of the area. The following methods to estimate the area of "viable" tumor were used:

1. Stringent: The percent of tumor occupied by areas that matched the cellularity and cytomorphology of the osteogenic component in the pre-treatment biopsy. This is the method routinely used at our institution for clinical reporting.

2. Inclusive: In addition to the areas measured by the stringent method, areas containing individual tumor cells, isolated atypical cells with smudged and clumped chromatin amid necrotic or fibrous stroma and/or tumor cartilage with intact, atypical chondrocytes were included in the total viable measurement.

Regardless of method, areas not counted as viable included (1) tumor osteoid that was acellular or contained only vessels; (2) coagulative necrosis; (3) hemorrhagic cystic change without atypical cells; and (4) sheets of foam cells [5, $16,18]$. The percent necrosis was then calculated as follows:

$\%$ necrosis $=\left(1-\frac{\text { sum of viable tumor cross sectional areas }}{\text { tumor gross cross sectional area }}\right) \times 100$

Treatment response was subsequently classified as good ( $\geq 90 \%$ necrosis) or poor $(<90 \%$ necrosis $)$ using both methods. Disease-free survival was calculated from the day of diagnosis until detection of recurrence or metastasis or most recent disease-free follow-up according to the KaplanMeier method and compared using the log-rank test. A pvalue of $<0.05$ was regarded as significant.

\section{RESULTS}

One hundred-six patients (68 males, 38 females) with a median age of 15 years (range 5-70) who received neoadjuvant chemotherapy were included (Table 1). Median clinical follow-up was 37 months (range 2 - 143). Ninetyfour percent of OS patients had primary lesions in the upper or lower extremity, with the remaining tumors located in the axial skeleton. The most commonly observed subtypes were osteoblastic $(n=58,55 \%)$ and chondroblastic $(n=25,24 \%)$. Disease progression was identified in 42 patients $(39 \%)$ 


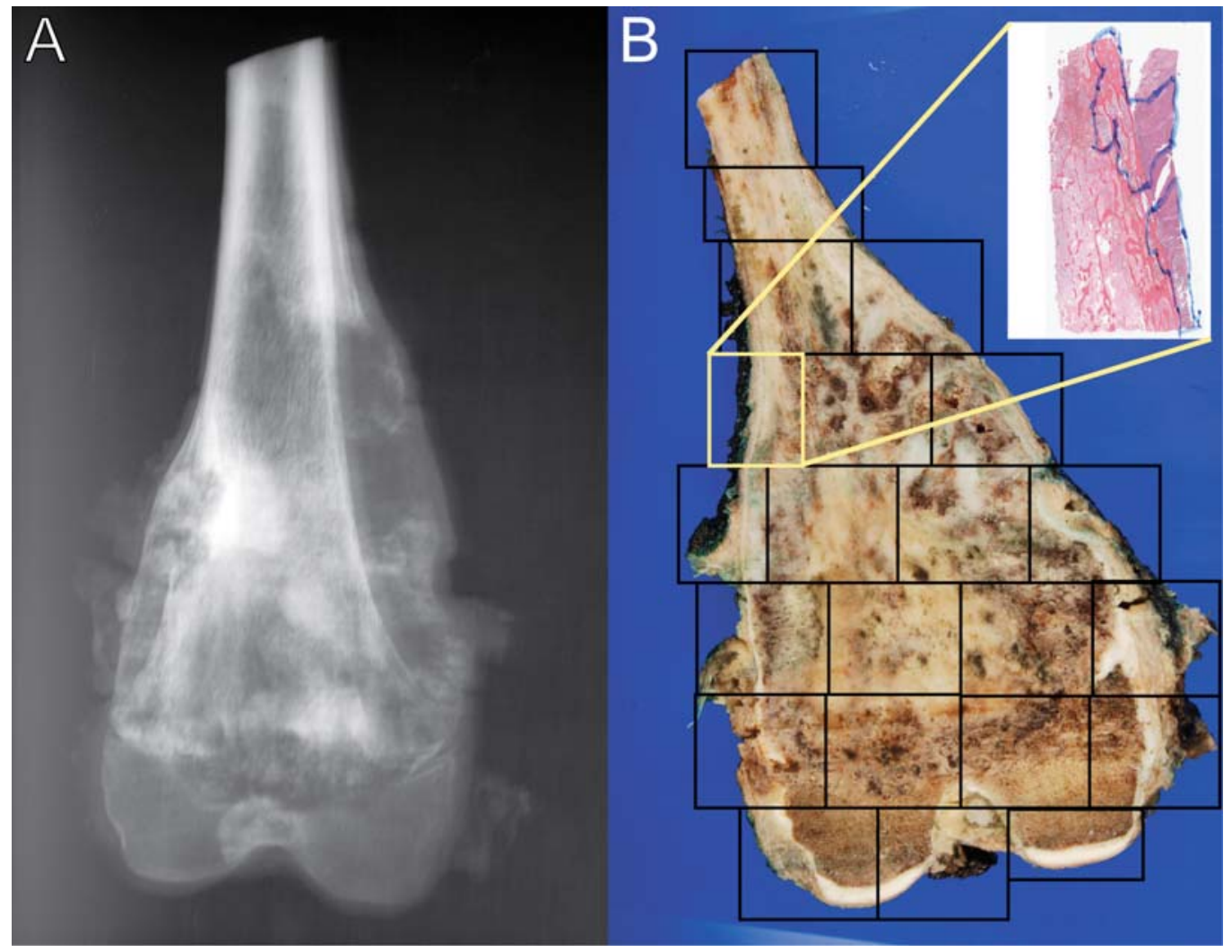

Fig. (1). Radiograph (A) and gross specimen (B) of a distal femoral osteosarcoma treated with neo-adjuvant chemotherapy. A full cross section is submitted for histologic evaluation as shown in (B). An example of how residual viable tumor is measured microscopically (B, inset).

Table 1. Summary of Clinical and Pathologic Characteristics of Osteosarcomas

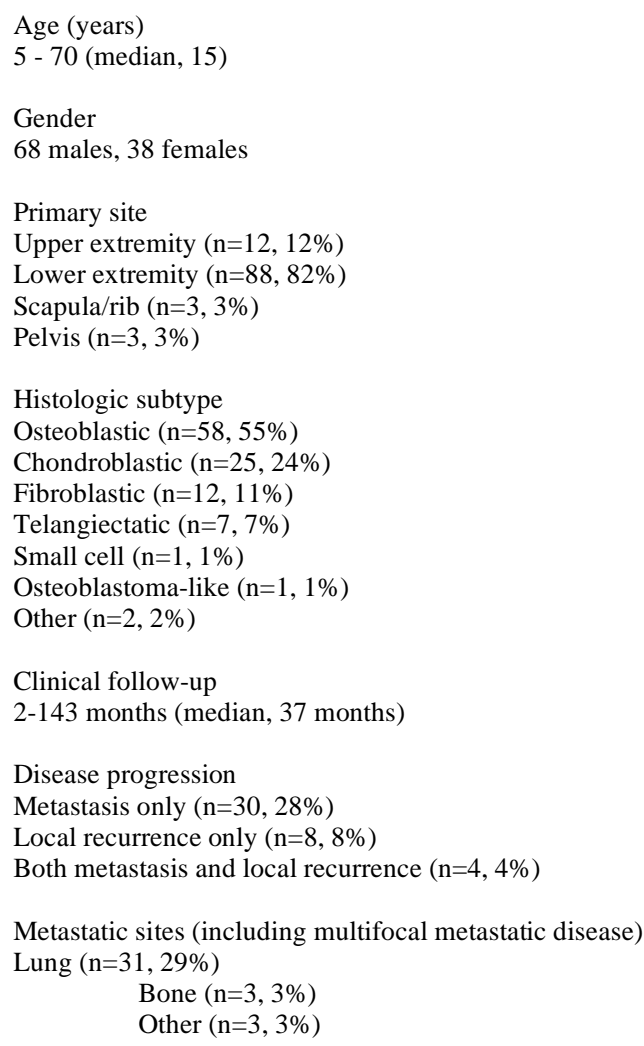

consisting of local recurrence, metastasis or both in $8(8 \%)$, $30(28 \%)$ and $4(3 \%)$ patients, respectively. Metastatic disease was present in 8 patients $(8 \%)$ at initial presentation. The average interval between biopsy and resection was 105 days (range 28 to 265 days) with the most common $(86 / 106$, $81 \%$ ) regimen consisting of MAP chemotherapy. The remaining patients received a variety protocols including methotrexate, doxorubicin and ifosfamide $(n=6,6 \%)$. doxorubicin and cisplatin $(n=5,5 \%)$ or methotrexate and doxorubicin $(n=3,4 \%)$.

Post-treatment histologic findings and the sorting of tumors based on chemotherapy response are outlined in Fig. (2). After neoadjuvant chemotherapy, 11 tumors (10\%) consisted only of areas of acellular tumor osteoid, fibrous stroma or coagulative necrosis but contained no viable tumor cells suggesting complete therapeutic response. These tumors were classified as good response in all methods.

Seventy-three tumors $(69 \%)$ contained at least one microscopic focus of OS indistinguishable from the corresponding pre-treatment biopsy but, of these, only 60 tumors $(47 \%)$ contained sufficient amounts of viable tumor to classify as poor response using the stringent method. Mitotic activity, including atypical forms, and nuclear pleomorphism were readily identified in these areas but osteoid and bone production was variable. One tumor showed rhabdoid cytomorphology after treatment. The highly cellular areas were distributed within the gross tumor either as a peripheral rim $(n=20,19 \%)$, discrete nodule(s) $(n=40,38 \%)$, or as a diffuse infiltrate $(n=13,12 \%)($ Fig. 3$)$. 


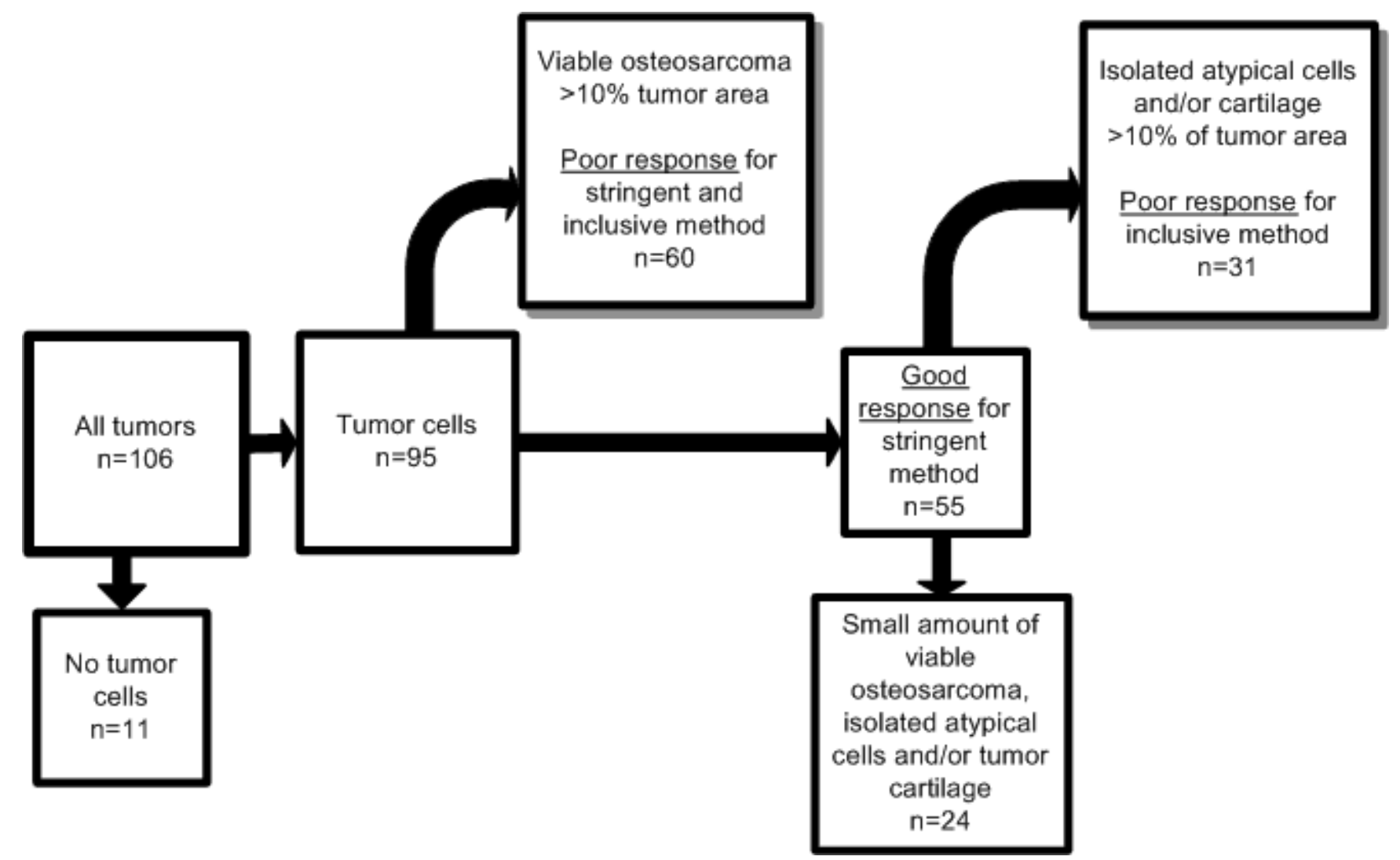

Fig. (2). Flow diagram of classification of neoadjuvant treated osteosarcomas based on histologic response. The curved arrows depict the subset of tumors classified as poor response by the stringent method and additional tumors reclassified into this subset using the inclusive method.

One tumor contained both a peripheral rim and multiple nodules of residual osteosarcoma.

In 35 tumors $(33 \%)$ we observed isolated tumor cells in necrotic or fibrotic stroma (Fig. 4). Some tumors contained both isolated cells and and sheets of pleomorphic osteosarcoma cells, but these two findings were usually discrete without transitional areas. Ten tumors $(8 \%)$ had residual tumor cartilage with viable, atypical chondrocytes occupying at least $50 \%$ of the lacunae one of which also had areas of isolated tumor cells. When including isolated tumor cells and cartilage, 31 tumors had sufficient such areas to reclassify them from good to poor response thus leaving only 25 tumors $(24 \%)$ in the good response category. Twentythree of the 31 patients $(74 \%)$ with reclassified tumors were disease-free at the end of the study. The median follow-up (49 months) and range of follow-up (2 - 130 months) of the patients with reclassified tumors were similar to the cohort as a whole. Furthermore, good chemotherapy response was statistically significant $(\mathrm{p}=0.008)$ to predict disease-free survival when measured using the stringent method but not when it was measured using the inclusive method $(\mathrm{p}=0.35$; Fig. 5) or when incorporating only tumor cartilage or only isolated atypical cells $(\mathrm{p}=0.1$ and $\mathrm{p}=0.22$, respectively; data not shown).

Additional histologic findings that were less commonly observed included sheets of foam cells $(n=5,5 \%)$ or hemorrhagic cystic changes $(n=3,3 \%)$ (Fig. 4).

\section{DISCUSSION}

The rationale for use of neoadjuvant therapy in the setting of osteosarcoma was set forth in a landmark study by Rosen et al. in 1976 [21]. A follow-up study elucidated the relationship between clinical outcome and the histopathology of the resection specimen [16]. The authors designed a grading scheme from no effect (grade I) to no histologic evidence of viable tumor (grade IV). Patients who achieved a grade III or IV response after neoadjuvant therapy had improved disease-free survival compared to those with a grade I or II response on follow-up.

While the microscopic assessment of response to neoadjuvant therapy has proven to be a good predictor of prognosis, guidance on determining the percent necrosis in these samples is somewhat incomplete and subjective. Picci et al. reported the most widely used method for the histologic evaluation of post-chemotherapy osteosarcoma specimens, illustrating a method for processing the gross specimen and determining the areas most likely to harbor residual viable tumor cells [5]. In their study, they noted the subjectivity in quantifying residual viable tumor and the inherent challenges of reproducibility with respect to the grading scheme established by Rosen et al. Therefore, they sought to broadly quantify the amount of necrosis, and used thresholds of $<50 \%, 50-80 \%$, and $>80 \%$ to determine response to therapy (poor, fair, and good, respectively), although a relationship between these response criteria and 

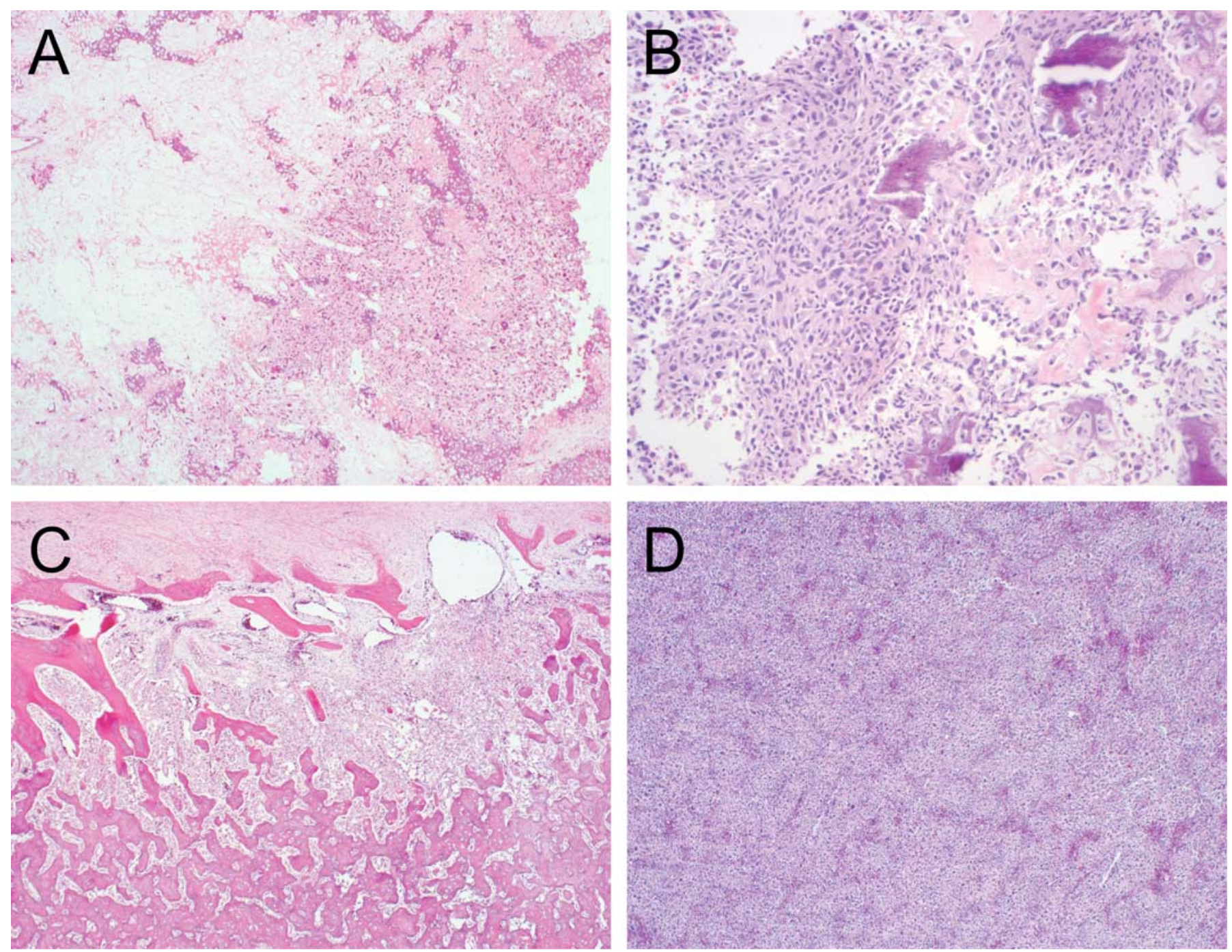

Fig. (3). Morphologic spectrum of residual, viable, osteosarcoma after neoadjuvant chemotherapy, H\&E stained sections. (A) Nodule of residual viable tumor (right half of image, $\mathrm{x} 40$ ); (B) At higher magnification, the tumor cells are essentially indistinguishable from untreated osteosarcoma (x200); (C) Peripheral rim of viable tumor between periosteum (top) and sclerotic bone without tumor cells (bottom, x40); (D) Diffuse pattern of viable tumor consisting of extensive sheets of tumor cells (x40).

clinical outcomes was not formally examined. Subsequent studies have attempted to quantify the response to neoadjuvant therapy, with $\geq 90 \%$ tumor cell necrosis typically, though not universally, used to define a "good" therapeutic response $[9,14,22]$.

In treated specimens, it is common to find areas containing widely dispersed or isolated atypical cells in necrotic, chemotherapy-responsive or fibrous stroma [18]. The density of these cells in any given area is usually low (< $5 \%$ of the surface area microscopically) and mitotic activity is conspicuously absent. It has been alternatively suggested that such a component represents persistent viable tumor, degenerating tumor, or reactive stromal elements such as osteoclasts [17-19]. Broad zones of tumor cartilage containing atypical cells are also occasionally present, most notably in treated chondroblastic osteosarcomas. While it may be reasonable to assume these areas can be ignored for the purpose of quantitating such questionably viable cells in total viable tumor either because of their uncertain biological potential or because their density is so low they are unlikely to be prognostically significant, we are unaware of any studies specifically supporting such an assumption. We addressed the clinical relevance of these morphologic findings by devising two separate methods to calculate percent tumor cell necrosis. The "stringent" method, routinely used at our institution, takes into account only areas with a high tumor cell density and mitotic activity that matches the pre-treatment biopsy. In the "inclusive" method, we also measured isolated atypical cells and tumor cartilage as viable. Using Kaplan-Meier survival analysis, and disease-free survival as outcome, the favorable predictive value of $\geq 90 \%$ necrosis loses its significance when considering isolated atypical cells and cellular tumor cartilage as viable. Whereas it remains unclear whether such areas constitute biologically "viable" sarcoma, the above data suggest that their presence does not, in fact, predict disease progression. When using the inclusive method to calculate percent necrosis, 31 tumors crossed over into the poor responder category. However, only a subset of these 

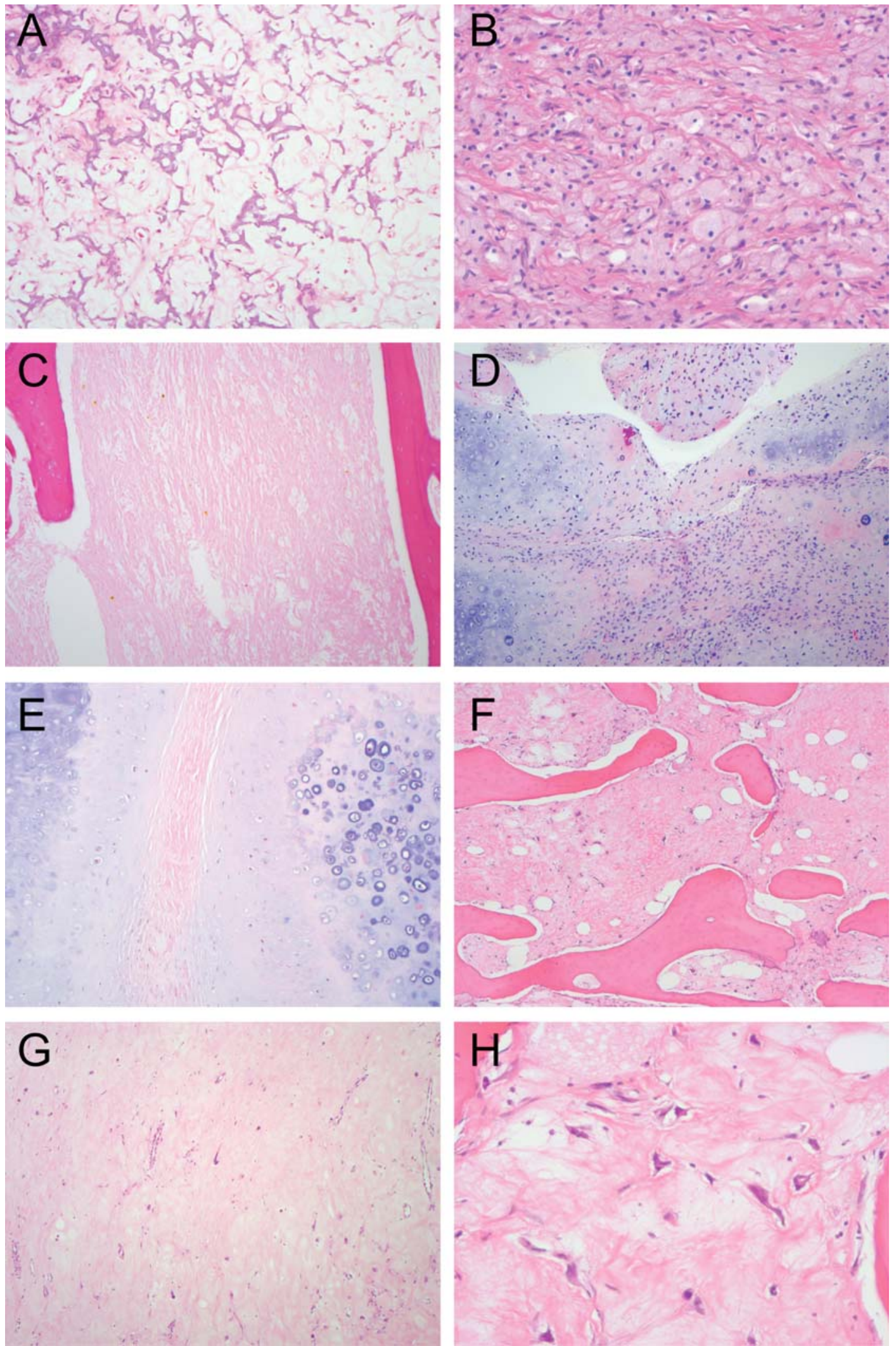

Fig. (4). Morphologic findings in osteosarcoma after neoadjuvant chemotherapy that do not predict a worse prognosis, H\&E stained sections. (A) Acellular tumor osteoid (x40); (B) Foam cells without atypia (x200); (C) Coagulative tumor necrosis (x40); (D) Pre-treatment chondroblastic osteosarcoma composed cartilaginous component mixed with more cellular, osteogenic, component (x100); (E) Posttreatment findings of same case as $\mathbf{C}$ showing persistence of cartilage but fibrosis replacing second component (x100). (F-H): Widely dispersed atypical cells in fibrous stroma (F, x40; G, x100; H, x400). 
A

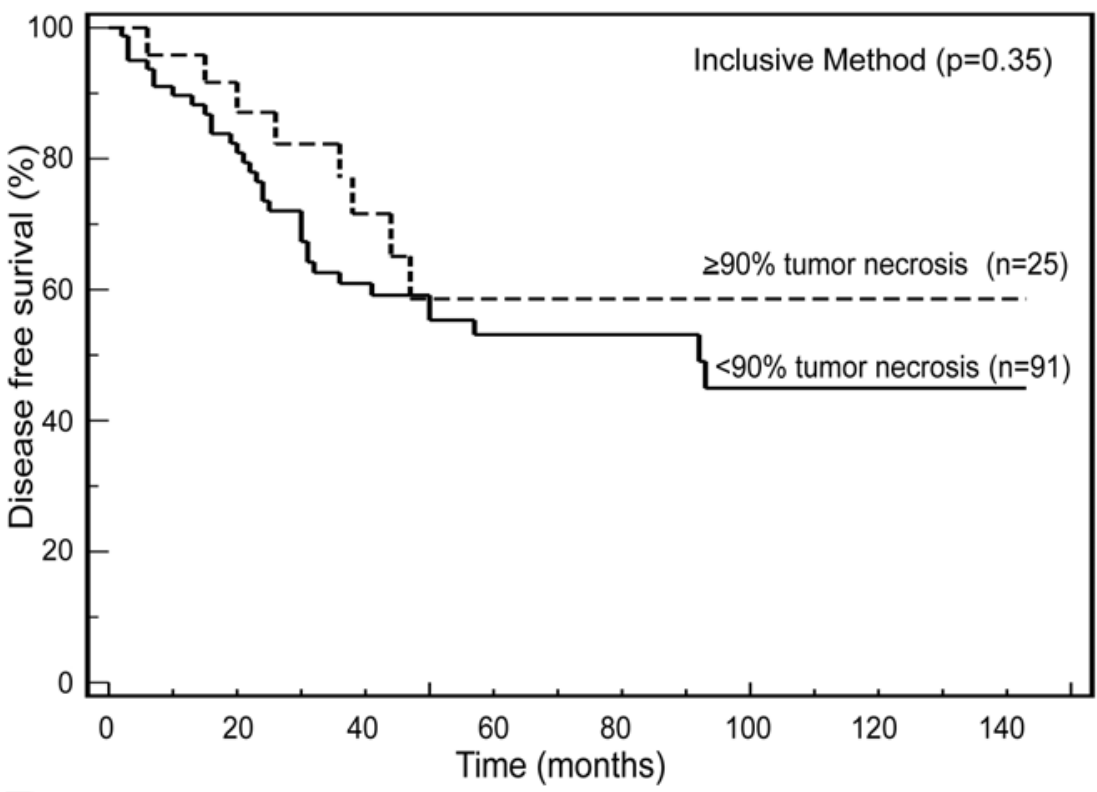

B

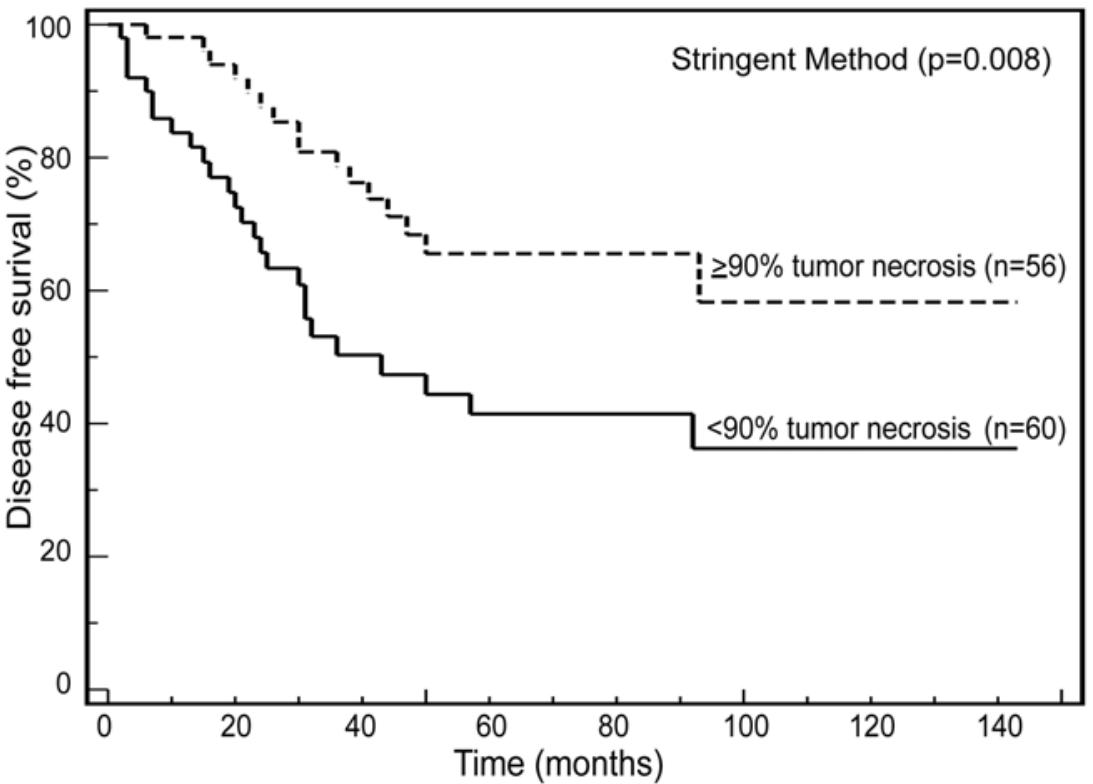

Fig. (5). Comparison of disease free survival as a function of $(\mathbf{A})$ inclusive $(\mathrm{p}=0.34)$ or $(\mathbf{B})$ stringent ( $\mathrm{p}=0.008)$ methods of calculating percent necrosis after neoadjuvant chemotherapy.

patients $(\mathrm{n}=8)$ had disease progression. We noted no pre- or post-therapy histologic features that distinguished the subgroup who did progress and the only clinical distinction was an overrepresentation of males $(7 / 8,88 \%)$ relative to the cohort as a whole $(68 / 106,64 \%)$ and relative to those who did not progress $(15 / 23,65 \%)$. However, gender did not appear to predict progression in the reclassified group $(\mathrm{p}=0.38$, Fisher's exact test $)$.

Several mechanisms may explain the reasons for the observed lack of clinical significance of isolated cells and tumor cartilage. Cells with "degenerative" atypia can be encountered in soft tissue sarcomas, where they can be quite numerous suggesting they represent neoplastic cells [23]. In treated OS, such cells are usually more sparse so their histotype is less clear $[17,18]$. In a few cases, we attempted to determine whether isolated atypical cells were osteoblastic, osteoclastic or other lineage using immunohistochemistry for skeletal differentiation markers previously reported by our group [24]. Immunohistochemistry was largely unsuccessful possibly as a result of the decalcification required to process these specimens (AEH, unpublished observations). In any event, the term "degenerative" implies that such cells lack the biological potential for continued proliferation, destructive growth and metastasis. The lack of mitoses in isolated cells supports this assertion, although we admit that it remains to be formally tested. Alternatively, isolated cells may retain malignant potential, but because of their low density, 
measuring areas with a few scattered cells as "viable," greatly overestimates the total volume of viable tumor cells. By analogy, although relatively broad zones of cartilage may be present, the effective volume of tumor cells, given the abundant extracellular matrix, may be overestimated when the area is measured. While it might be possible, for example using computerized image analysis to accurately measure the contribution to total area made by cells, this is impractical for routine practice. Furthermore, since osteosarcoma is by definition a matrix-producing tumor, even highly cellular areas with no obvious treatment effect contain osteoid or bone and yet stroma is not excluded in the measurement of these areas $[8,12,14,15]$. We submit, based on the loss of prognostic significance when including isolated cells and/or cartilage, that these areas should be ignored when quantitating viable tumor. Interestingly, previous reports have observed that chondroblastic osteosarcoma has a better prognosis than might be expected given usually poor chemotherapy response [2, 25]. Our results provide an explanation for this discrepancy. Namely, residual tumor cartilage may not be prognostically significant when measuring chemoresponsiveness.

Frequently, the areas of residual viable tumor formed a rim or multiple irregularly shaped nodules and the tumor itself, grossly, usually approximated an irregular ellipse. Further, evaluating only a single slice of a treated osteosarcoma microscopically has the potential to miss macroscopic areas of viable tumor out of the sampled plane. Although the exact cross sectional area for irregular shapes can be calculated using more complex geometric formulas or digital image-capture techniques, and additional slices of tumor can be sampled, our data show that such laborious procedures are unnecessary for routine use. Rather, simply estimating areas (microscopically viable and gross cross section) using the product of maximum length and maximum width results in statistically significant correlation with outcome. That percent necrosis remains statistically significant as a prognostic marker, despite the estimates described above, suggests the $\geq 90 \%$ threshold for "good" response is a very robust measure.

The present study was not intended to test the underlying causes for variations in chemotherapy response between tumors, whether from differences in therapy or tumor biology [22, 26-28]. Although most patients in this study received MAP, many participated in randomized trials which included adjuvant agents such as ifosfamide or interferon. One important confounder regarding chemotherapy was considered. Namely, could the choice of adjuvant chemotherapy (e.g. addition of ifosfamide) explain our results? That is, if more aggressive adjuvant therapy was given only to patients with "poor" response by considering residual cartilage or isolated atypical cells (inclusive criteria) as viable, then treatment may have improved their diseasefree survival by effectively simulating that they were miscategorized as "poor" responders. Although this could potentially impact our analysis, recall that the "stringent" criteria were used at our institution to classify chemotherapy response for clinical purposes. Consequently, adjuvant treatment decisions were not based on the presence or absence of residual tumor cartilage or isolated atypical cells. Conversely, patients judged as "poor" response using the stringent criteria were treated more aggressively typically with ifosfamine and etoposide added to the postoperative regimen.

The group of patients, tumors reclassified from good to poor response by the inclusive method had a similar range and mean follow-up time to the cohort of patients in this study as a whole arguing against attrition bias as a confounder in this group.

\section{CONCLUSION}

In summary, the purpose of this study was to describe the patterns of histologic response in OS resection specimens and determine the prognostic significance, if any, in including isolated atypical cells and tumor cartilage. Here we have addressed important issues related to processing and assessing neoadjuvant-treated OS resection specimens from a large cohort of patients at a single institution. This study validates that percent tumor necrosis after chemotherapy, when correctly measured, is an important prognostic indicator. Our results suggest that isolated atypical cells and tumor cartilage should be excluded when measuring viable tumor although we recognize that larger, multi-institutional, studies may be desirable to further validate this recommendation.
ABBREVIATIONS
OS = Osteosarcoma
MAP = Methotrexate, doxorubicin, , cisplatin

\section{CONFLICT OF INTEREST}

The authors confirm that this article content has no conflict of interest.

\section{ACKNOWLEDGEMENTS}

Declared none.

\section{REFERENCES}

[1] Mirabello L, Troisi RJ, Savage SA. Osteosarcoma incidence and survival rates from 1973 to 2004: data from the Surveillance, Epidemiology, and End Results Program. Cancer 2009; 115: 153143.

[2] Hauben EI, Weeden S, Pringle J, Van Marck EA, Hogendoorn PC. Does the histological subtype of high-grade central osteosarcoma influence the response to treatment with chemotherapy and does it affect overall survival? A study on 570 patients of two consecutive trials of the European Osteosarcoma Intergroup. Eur J Cancer 2002; 38: 1218-25.

[3] Huvos AG, Rosen G, Marcove RC. Primary osteogenic sarcoma: pathologic aspects in 20 patients after treatment with chemotherapy en bloc resection, and prosthetic bone replacement. Arch Pathol Lab Med 1977; 101: 14-8.

[4] Bacci G, Picci P, Ferrari S, et al. Primary chemotherapy and delayed surgery for nonmetastatic osteosarcoma of the extremities. Results in 164 patients preoperatively treated with high doses of methotrexate followed by cisplatin and doxorubicin. Cancer 1993; 72: 3227-38.

[5] Picci P, Bacci G, Campanacci M, et al. Histologic evaluation of necrosis in osteosarcoma induced by chemotherapy. Regional mapping of viable and nonviable tumor. Cancer 1985; 56: 1515-21.

[6] Bielack S, Carrle D, Casali PG. Osteosarcoma: ESMO clinical recommendations for diagnosis, treatment and follow-up. Ann Oncol 2009; 20 Suppl 4: 137-9.

[7] Geller DS, Gorlick R. Osteosarcoma: a review of diagnosis, management, and treatment strategies. Clin Adv Hematol Oncol 2010; 8: 705-18.

[8] Huvos AG. Bone tumors: diagnosis, treatment and prognosis. $2^{\text {nd }}$ ed. Philadelphia: WB Saunders 1991. 
[9] Bacci G, Mercuri M, Longhi A, et al. P. Grade of chemotherapyinduced necrosis as a predictor of local and systemic control in 881 patients with non-metastatic osteosarcoma of the extremities treated with neoadjuvant chemotherapy in a single institution. Eur J Cancer 2005; 41: 2079-85.

[10] Hudson M, Jaffe MR, Jaffe N, et al. Pediatric osteosarcoma: therapeutic strategies, results, and prognostic factors derived from a 10-year experience. J Clin Oncol 1990; 8: 1988-97.

[11] Provisor AJ, Ettinger LJ, Nachman JB, et al. Treatment of nonmetastatic osteosarcoma of the extremity with preoperative and postoperative chemotherapy: a report from the Children's Cancer Group. J Clin Oncol 1997; 15: 76-84.

[12] Rosen G, Caparros B, Huvos AG, et al. Preoperative chemotherapy for osteogenic sarcoma: selection of postoperative adjuvant chemotherapy based on the response of the primary tumor to preoperative chemotherapy. Cancer 1982; 49: 1221-30.

[13] Winkler K, Beron G, Delling G, et al. Neoadjuvant chemotherapy of osteosarcoma: results of a randomized cooperative trial (COSS82) with salvage chemotherapy based on histological tumor response. J Clin Oncol 1988; 6: 329-37.

[14] Raymond AK, Chawla SP, Carrasco CH, et al. Osteosarcoma chemotherapy effect: a prognostic factor. Semin Diagn Pathol 1987; 4: 212-36.

[15] Wold LE. Practical approach to processing osteosarcomas in the surgical pathology laboratory. Pediatr Dev Pathol 1998; 1: 449-54.

[16] Rosen G, Marcove RC, Caparros B, Nirenberg A, Kosloff C, Huvos AG. Primary osteogenic sarcoma: the rationale for preoperative chemotherapy and delayed surgery. Cancer 1979; 43: 2163-77.

[17] Coffin CM, Lowichik A, Zhou H. Treatment effects in pediatric soft tissue and bone tumors: practical considerations for the pathologist. Am J Clin Pathol 2005; 123: 75-90.

[18] Min HS, Kang HG, Ro JY. Therapy Related Changes in Osteosarcoma and Ewing Sarcoma of Bone. Open Pathol J 2009; 3: 99-105.

[19] Rosenberg AE, Cleton-Jansen AM, de Pinieux G, Deyrup AT, Hauben E, Squire J, Conventional osteosarcoma. In: Fletcher, CD,
Bridge JA, Hogendoorn PC, Mertens F, Eds. WHO Classification of tumours of soft tissue and bone. $4^{\text {th }} \mathrm{ed}$. Lyons, France: IARC Press 2013; pp. 282-8.

[20] Mangham DC, Athanasou NA. Guidelines for histopathological specimen examination and diagnostic reporting of primary bone tumours. Clin Sarcoma Res 2011; 1: 6.

[21] Rosen G, Murphy ML, Huvos AG, Gutierrez M, Marcove RC. Chemotherapy, en bloc resection, and prosthetic bone replacement in the treatment of osteogenic sarcoma. Cancer 1976; 37: 1-11.

[22] Ferrari S, Zanella L, Alberghini M, Palmerini E, Staals E, Bacchini P. Prognostic significance of immunohistochemical expression of ezrin in non-metastatic high-grade osteosarcoma. Pediatr Blood Cancer 2008; 50: 752-6.

[23] Lucas DR, Kshirsagar MP, Biermann JS, et al. Histologic alterations from neoadjuvant chemotherapy in high-grade extremity soft tissue sarcoma: clinicopathological correlation. Oncologist 2008; 13: 451-8.

[24] Horvai AE, Roy R, Borys D, O'Donnell RJ. Regulators of skeletal development: a cluster analysis of 206 bone tumors reveals diagnostically useful markers. Mod Pathol 2012; 25: 1452-61.

[25] Bacci G, Forni C, Ferrari S, et al. Neoadjuvant chemotherapy for osteosarcoma of the extremity: intensification of preoperative treatment does not increase the rate of good histologic response to the primary tumor or improve the final outcome. J Pediatr Hematol Oncol 2003; 25: 845-53.

[26] Borys D, Canter RJ, Hoch B, et al. P16 expression predicts necrotic response among patients with osteosarcoma receiving neoadjuvant chemotherapy. Hum Pathol 2012; 43: 1948-54.

[27] Park HS, Cho SG, Kim CK, et al. Heat shock protein hsp72 is a negative regulator of apoptosis signal-regulating kinase 1 . Mol Cell Biol 2002; 22: 7721-30.

[28] Trieb K, Lechleitner T, Lang S, Windhager R, Kotz R, Dirnhofer S. Heat shock protein 72 expression in osteosarcomas correlates with good response to neoadjuvant chemotherapy. Hum Pathol 1998; 29: $1050-5$.

(C) Cascio et al.; Licensee Bentham Open.

This is an open access article licensed under the terms of the Creative Commons Attribution Non-Commercial License (http://creativecommons.org/licenses/by$\mathrm{nc} / 3.0 /$ ) which permits unrestricted, non-commercial use, distribution and reproduction in any medium, provided the work is properly cited. 